\title{
REVISITING THE CHRIST-KISELEV'S MULTI-LINEAR OPERATOR TECHNIQUE AND ITS APPLICATIONS TO SCHRÖDINGER OPERATORS
}

\author{
WENCAI LIU
}

\begin{abstract}
We established a generalized version of the Christ-Kiselev's multi-linear operator technique to deal with the spectral theory of Schrödinger operators. As applications, several spectral results of perturbed periodic Schrödinger operators are obtained, including WKB solutions, sharp transitions of preservation of absolutely continuous spectra, criteria of absence of singular spectra and sharp bounds of the Hausdorff dimension of singular spectra.
\end{abstract}

\section{INTRODUCTION AND MAIN RESULTS}

Stimulated by several problems posted by Barry Simon at ICMP in 1994 and 2000 [38], the theory of Schrödinger operators with decaying potentials have been made significant progress in the past 25 years through the work of Christ, Deift, Denisov, Killip, Kiselev, Last, Molchanov, Remling, Simon, Stolz and among others. We refer readers to two survey articles [6, 11] and references therein for details.

One of the central topics in the area is to understand the criteria of preservation of absolutely continuous spectra under $L^{p}$ or power decaying perturbations. The onedimensional case of free Schrödginer operators is now well understood mainly by two approaches. The first one is to study the spectral theory of Schrödinger operators via establishing the WKB type eigensolutions. Another celebrated approach starting with Deift and Killip used a completely different idea: sum rules. The sum rule approach can handle the critical case $p=2$, which was missing by WKB methods, but less understandings about the dynamics.

The research by the first approach (WKB method) has been achieved a great success by Christ, Kiselev and Remling [1-4, 13, 14, 32]. In [2- 4] $]$, Christ and Kiselev developed a way, referred to as multi-linear operator techniques, to deal with the WKB type solutions, which turned out to be a robust approach. In this paper, we will establish a more general version of the Christ-Kiselev's multi-linear operator technique than that appearing in 2 24] (see Section 22). We added several significant technical ingredients in the proof, which allows us to strength several conclusions with less assumptions. For example, we proved the conclusion in Lemma 2.8 as it was appearing in [3, 4, 12] without lower bound assumptions on second and third derivatives (see (27)). As applications, we obtained several new important results. We will talk about the details later. Our proofs are self-contained except for several well known facts, such as ChristKiselev Lemma (Lemma 2.7) and multiplicative bounds (Theorem 2.1). We expect our 
new version will serve as a good resource for readers to understand Christ-Kiselev's machinery and as well as lead to more applications in the future.

Multi-linear operator techniques to establish the WKB type eigensolution are based on writing down the differential equation in an integral form and seeking a formal series solution. Each individual term of the series is defined by a multi-integral operator. The difficulty lies in giving rigorous definition of improper integrals in a suitable topology, showing the convergence of the series in a proper measure space, and verifying that the formal series solution is an actual solution. The main scheme of our proofs is definitely developed from Christ-Kiselev. However, several important technical improvements have been added to Christ-Kiselev's scheme. The first new ingredient we want to highlight is that we define the multi-linear operators as iterations, which significantly simplifies the arguments. This is very different from Christ-Kiselev's plan (see the proof of Lemma 4.2 in [4]). Our approach is more natural and will make it much easier to verify that the formal series solution is an actual solution. The price we need to pay is to show the existence of a stronger limit in the definition of multi-integral operators. See Remark 2.6. Another new important improvement we want to mention is that we will give two ways to establish the WKB type solution. One way closely follows from the Christ-Kiselev's approach. Our new approach allows us to avoid using maximal operators and simplify the original approach of Christ and Kiselev. See Section 5. Our new proof is based on modifications of norms of a family of Banach spaces.

Finally, we want to highlight several great discoveries in our applications, which we believe to be of independent interest. We obtain a nice formula of the spectral measure of eventually periodic operators by the combination of Floquet theory with Weyl theory. See Section 7 . It possibly provides more opportunities to use approximations to investigate the spectral theory of perturbed periodic Schrödinger operators. We also obtain sharp bounds of a family of oscillatory integrations, which are originally arising from eigen-equations. A simple case of oscillatory integrations here comes from studying Schrödinger operators with Wigner-von Neumann type potentials [42]. Wigner-von Neumann type functions are fundamental generators to construct (finitely many or countably many)embedded eigenvalues dating back to Simon [37] and receive continuing attentions [8, 15, 24 27]. Our sharp bounds of oscillatory integrations are generalizations and quantitative versions of all previous statements in the area [8, 15, 22 25, 37]. The novelty here is to split frequencies into higher and lower ones so that different tools can be used. See explanations after Corollary 1.7.

Let us move to applications. We will investigate the spectral theory of the onedimensional perturbed periodic Schrödinger operator, namely,

$$
H u=-u^{\prime \prime}+\left(V(x)+V_{0}(x)\right) u,
$$

where $V_{0}(x)$ is 1-periodic and $V(x)$ is a decaying perturbation.

When $V \equiv 0$, we have a 1-periodic Schrödinger operator,

$$
H_{0} u=-u^{\prime \prime}+V_{0} u \text {. }
$$

We are interested in spectral transitions of operators (1) under decaying perturbations $V$. The sharp transition for (dense) embedded eigenvalues was recently obtained 
by the author and Ong [25] with some partial results in the past [17, 20, 28, 30, 35]. Sharp transitions of preservation of absolutely continuous spectra and sharp bounds of Hausdorff dimensions of singular spectra will be obtained as applications of Theorem 2.5 .

For simplicity, we only consider the equation on the half line $\mathbb{R}^{+}$. All the results can be generalized to the whole line $\mathbb{R}$.

Denote by $\ell^{p}\left(L^{1}\right)\left(\mathbb{R}^{+}\right)$the Banach space of all measurable functions from $\mathbb{R}^{+}$to $\mathbb{R}$ with the norm

$$
\|f\|_{\ell^{p}\left(L^{1}\right)}=\left(\sum_{k=0}^{\infty}\left(\int_{k}^{k+1}|f(x)| d x\right)^{p}\right)^{1 / p} .
$$

This Banach space contains $L^{1}+L^{p}$. If $p \leq q$, then $\ell^{p}\left(L^{1}\right) \subset \ell^{q}\left(L^{1}\right)$. For simplicity, $V_{0}$ is always assumed to be in $L^{1}[0,1]$ and periodic.

Let $S=\cup_{n=0}^{\infty}\left(a_{n}, b_{n}\right)$ be the band spectrum of the operator given by (2) and $\varphi(x, E)$ be the Floquet solution for $E \in S$. By Theorem 2.5 and additional spectral analysis, we have following two theorems.

Theorem 1.1. If the potential $V \in \ell^{p}\left(L^{1}\right)$ for some $1 \leq p<2$, then the set $S$ is an essential support of the absolutely continuous spectrum of the operator $H=H_{0}+V$ with any boundary condition at zero. Moreover, for a.e. $E \in S$, there exists solution $u(x, E)$ of the equation

$$
-u^{\prime \prime}+\left(V_{0}(x)+V(x)\right) u=E u
$$

with the asymptotical behavior

$$
u(x, E)=\varphi(x, E) \exp \left(\frac{i}{2 \Im\left(\varphi \bar{\varphi}^{\prime}\right)} \int_{0}^{x} V(t)\left|\varphi^{2}(t, E)\right| d t\right)(1+o(1))
$$

as $x \rightarrow \infty$.

Let $p^{\prime}$ be the conjugate number to $p$, namely $\frac{p}{p-1}$ for $p>1$.

Theorem 1.2. Suppose the potential $|x|^{\gamma} V \in \ell^{p}\left(L^{1}\right)$ for some $1<p \leq 2, \gamma>0$ with $\gamma p^{\prime} \leq 1$. Then for every $E \in S$, there exists solution $u(x, E)$ of $H u=E u$ satisfying the asymptotical behavior (4), except for a set of values of $E$ in $S$ with Hausdorff dimension less or equal than $1-\gamma p^{\prime}$.

As a corollary, we have

Corollary 1.3. Suppose $V(x)=\frac{O(1)}{1+x^{\alpha}}$ with $\alpha \in[0,1]$. Then for every $E \in S$, there exists solution $u(x, E)$ of $H u=E u$ satisfying the asymptotical behavior (4), except for a set of values of $E$ in $S$ with Hausdorff dimension less or equal than $2(1-\alpha)$.

Remark 1.4. It has been shown that the multilinear operator technique is not extendable to tackle the case $p=2$ by Muscalu-Tao-Thiele [29].

If $V_{0} \equiv 0$, Theorems 1.1, 1.2 and Corollary 1.3 have been proved in [2 4, 33]. If $V_{0} \equiv 0$, Remling 34] and Kriecherbauer-Remling [18] constructed examples which show that $2(1-\alpha)$ in Corollary 1.3 is the best bound to be achieved. Under a stronger 
assumption of potentials $V$, that is $|x|^{\gamma} V \in \ell^{p}\left(L^{1}\right)$ for some $\gamma>0$, Theorem 1.1 has been proved by Christ and Kiselev [1]. For $p>2$, it is known that Theorem [1.1 is not true even for the case $V_{0} \equiv 0$ (see [16] for example). For $p=2$, the first part of Theorem 1.1 is proved by Deift-Killip for the case $V_{0} \equiv 0$ [5] and Killip [10] by different approaches. For $p=2$, the second part is open even for $V_{0} \equiv 0$.

Our another interest in this paper is to investigate spectral transitions for singular spectra. Let us review the results for the case $V_{0} \equiv 0$ first.

I: If $V(x)=\frac{o(1)}{1+x}, H_{0}+V$ does not have any positive eigenvalues [9].

II: Wigner-von Neumann type functions imply that there exist potentials $V(x)=$ $\frac{O(1)}{1+x}$ such that $H_{0}+V$ has positive eigenvalues [42].

III: For any given positive function $h(x)$ tending to infinity as $x \rightarrow \infty$, there exist potentials $V(x)$ such that $|V(x)| \leq \frac{h(x)}{1+x}$ and operators $H_{0}+V$ have dense embedded eigenvalues [31, 37].

IV: If $V(x)=\frac{O(1)}{1+x}, H_{0}+V$ does not have the singular continuous spectrum [15]. V: For any given any positive function $h(x)$ tending to infinity as $x \rightarrow \infty$, there exist potentials $V(x)$ such that $|V(x)| \leq \frac{h(x)}{1+x}$ and singular continuous spectra of operators $H_{0}+V$ are non-empty [15].

Clearly, the above statements from I to $\mathrm{V}$ imply the criteria for the absence of singular spectra (eigenvalues and singular continuous spectra). It is natural to expect that corresponding criteria are true for any non-zero periodic function $V_{0}$. For embedded eigenvalues, cases I, II and III have been proved to be true for general $V_{0}$ [17, 25]. For the singular continuous spectrum, we conjecture that IV and V hold for general periodic functions $V_{0}$. In this paper, we prove half of the conjecture.

Theorem 1.5. Suppose $V(x)=\frac{O(1)}{1+x}$. Then the singular continuous spectrum of $H=H_{0}+V$ with any boundary condition is empty.

Theorem 1.5 and case I for general $V_{0}$ imply

Corollary 1.6. Suppose $V(x)=\frac{o(1)}{1+x}$. Then the spectral measure of $H_{0}+V$ with any boundary condition at zero is purely absolutely continuous in $S$.

Define $P$ as

$$
P=\left\{E \in \mathbb{R}:-u^{\prime \prime}+\left(V(x)+V_{0}(x)\right) u=E u \text { has an } L^{2}\left(\mathbb{R}^{+}\right) \text {solution }\right\},
$$

It has been proved that $P \cap S$ is a countable set provided $V(x)=\frac{O(1)}{1+x}[23]$. Therefore, Theorem 1.5 also implies

Corollary 1.7. Suppose $V(x)=\frac{O(1)}{1+x}$. Then except for countably many boundary conditions at zero, the spectral measure of $H_{0}+V$ is purely absolutely continuous in $S$.

The proof of Theorem 1.5] is inspired by [15], where the case $V_{0} \equiv 0$ has been proved. Under the assumption of Theorem 1.5, the singular component of the spectral measure is supported on a set of zero Hausdoff dimension by Corollary 1.3. Following the strategy in [15], additional four steps are needed. Step 1: establish the quantitatively 
almost orthogonality among Prüfer angles. Step 2: control the total number of "separate energies"1 based on step 1. Step 3: establish the spectral measure of Schrödinger operators with eventually zero potentials. Step 4: use the spectral measure of eventually zero potentials to do approximations.

In our case, the periodic potentials are involved in so that the problem is a lot more complicated, in particular, the step 1 and step 3. Let us mention that the almost orthogonality is between $\frac{\theta\left(x, E_{1}\right)}{1+x}$ and $\frac{\theta\left(x, E_{2}\right)}{1+x}$ in Hilbert space $L^{2}([0, B],(1+$ $x) d x$ ) for large $B$, where $\theta\left(x, E_{1}\right)$ (resp. $\theta\left(x, E_{2}\right)$ ) is the (generalized) Prüfer angle with respect to energy $E_{1}$ (resp. $E_{2}$ ). In [15], Kiselev established sharp bounds of the almost orthogonality of perturbed free Schrödinger operators (step 1). For our cases, rather than using the standard Prüfer variables, we have to instead use the generalized Prüfer variables, which is known to be difficult to handle. The almost orthogonality of general cases was proved very recently in [25] without quantitative estimates, which is used to construct embedded eigenvalues. However, in order to deal with the singular continuous spectrum, the quantitative bounds are essential, in particular, we need to control the blowup when $E_{1}$ approaches to $E_{2}$. In [25], one of the innovations is the use of Fourier expansions to ensure that some key terms decay sufficiently quickly. For the rest of terms, it can be controlled by using oscillatory integral techniques to establish the well cancellation between positive and negative parts of the integrals. Even through we use Fourier expansions and oscillatory integral techniques from [25] in a quantitative way, the bounds are not enough. We overcome the difficulty by splitting the frequencies into high and low ones, where the frequency comes from the quasimomentum of Floquet theory. For high frequencies, we quantify Fourier expansions and oscillatory integral techniques in [25] in a sharp way. For low frequencies, we combine Fourier expansions in [25] with the techniques in [15] to establish the sharp bounds.

In the end, we remark that the spectral theory of perturbed periodic operators in higher dimensions is much more difficult. We refer readers to [19] for details.

\section{Christ-Kiselev's multi-linear operator techniques}

Since we only consider operators on the half line $\mathbb{R}_{+}$, all the functions are defined on $\mathbb{R}_{+}$. Let us introduce the multilinear operator $M_{n}$, acting on $n$ functions $g_{k}$, $k=1,2, \cdots, n$, by

$$
\begin{aligned}
M_{n}\left(g_{1}, g_{2}, \cdots, g_{n}\right)\left(x, x^{\prime}\right) & =\int_{x \leq t_{1} \leq \cdots \leq t_{n} \leq x^{\prime}} \prod_{k=1}^{n} g_{k}\left(t_{k}\right) d t_{k} \\
& =\int_{x \leq t_{1} \leq \cdots \leq t_{n}<\infty} \prod_{k=1}^{n} g_{k}\left(t_{k}\right) \chi_{\left[0, x^{\prime}\right]}\left(t_{k}\right) d t_{k},
\end{aligned}
$$

where $\chi$ is the characteristic function. If there is a single function $g$ such that $g_{k}$ is in $\{g, \bar{g}\}$, we write it down by $M_{n}(g)\left(x, x^{\prime}\right)$.

A collection of subintervals $E_{j}^{m} \subset \mathbb{R}_{+}, 1 \leq j \leq 2^{m}$ and $m \in \mathbb{Z}^{+}$[4] is called a martingale structure if the following is true:

\footnotetext{
${ }^{1}$ See the definition in Section 8
} 
- $\mathbb{R}_{+}=\cup_{j} E_{j}^{m}$ for every $m$.

- $E_{j}^{m} \cap E_{i}^{m}=\emptyset$ for every $i \neq j$.

- If $i<j, x \in E_{i}^{m}$ and $x^{\prime} \in E_{j}^{m}$, then $x<x^{\prime}$.

- For every $m, E_{j}^{m}=E_{2 j-1}^{m+1} \cup E_{2 j}^{m+1}$.

Denote by $\chi_{j}^{m}=\chi_{E_{j}^{m}}$. Let $\mathfrak{B}_{s}$ be the Banach space consisting of all complex-valued sequences $a=a(m, j)$ indexed by $1 \leq m<\infty$ and $1 \leq j \leq 2^{m}$, for which

$$
\|a\|_{\mathfrak{B}_{s}}=\sum_{m \in \mathbb{Z}_{+}} m^{s}\left(\sum_{j=1}^{2^{m}}|a(m, j)|^{2}\right)^{1 / 2}<\infty .
$$

Denote by $\mathfrak{B}=\mathfrak{B}_{1}$. For any function $g$ on $\mathbb{R}_{+}$, we can define a sequence with index $m \in \mathbb{Z}_{+}$and $1 \leq j \leq 2^{m}$,

$$
\left\{\int_{E_{j}^{m}} g(x) d x\right\}=\left\{\int_{\mathbb{R}_{+}} g(x) \chi_{j}^{m} d x\right\} .
$$

By abusing the notation, denote by

$$
\|g\|_{\mathfrak{B}^{s}}=\left\|\left\{\int_{E_{j}^{m}} g(x) d x\right\}\right\|_{\mathfrak{B}^{s}}=\sum_{m}^{\infty} m^{s}\left(\sum_{j=1}^{2^{m}}\left|\int_{E_{j}^{m}} g(x) d x\right|^{2}\right)^{\frac{1}{2}} .
$$

Define

$$
M_{n}^{*}\left(g_{1}, g_{2}, \cdots, g_{n}\right)=\sup _{0<x \leq x^{\prime}<\infty}\left|M_{n}\left(g_{1}, g_{2}, \cdots, g_{n}\right)\left(x, x^{\prime}\right)\right|
$$

and

$$
M_{n}^{*}(g)=\sup _{0<x \leq x^{\prime}<\infty}\left|M_{n}(g)\left(x, x^{\prime}\right)\right| .
$$

Theorem 2.1. [G] For any martingale structure $E_{j}^{m} \subset \mathbb{R}_{+}, 1 \leq j \leq 2^{m}$ and $m \in \mathbb{Z}^{+}$, the following estimates hold,

$$
M_{n}^{*}\left(g_{1}, g_{2}, \cdots, g_{n}\right) \leq C^{n} \prod_{i=1}^{n}\left\|g_{i}\right\|_{\mathfrak{B}},
$$

and

$$
M_{n}^{*}(g) \leq C^{n} \frac{\|g\|_{\mathfrak{B}}^{n}}{\sqrt{n !}}
$$

where $C$ is an absolute constant.

A martingale structure $E_{j}^{m} \subset \mathbb{R}_{+}, 1 \leq j \leq 2^{m}$ and $m \in \mathbb{Z}^{+}$is said to be adapted in $\ell^{p}\left(L^{1}\right)$ to a function $f$ if for all possible $m, j$,

$$
\left\|f \chi_{j}^{m}\right\|_{\ell^{p}\left(L^{1}\right)}^{p} \leq 2^{-m}\|f\|_{\ell^{p}\left(L^{1}\right)}^{p} .
$$

Since all the functions are in $\ell^{p}\left(L^{1}\right)$, we omit "adapted" in the rest of this paper.

Lemma 2.2. (p.433, [4]) For any function $f \in \ell^{p}\left(L^{1}\right)$, there exists a martingale structure $\left\{E_{j}^{m} \subset \mathbb{R}_{+}: m \in \mathbb{Z}_{+}, 1 \leq j \leq 2^{m}\right\}$ to $f$. 
Suppose $P$ is a linear or sublinear operator: for any function $f$ on $\mathbb{R}_{+}, P(f)(\lambda)$ is a function on a interval $J \subset \mathbb{R}$. For $s>0$, denote by

$$
\begin{aligned}
G_{P(f)(\lambda)}^{(s)} & =\left\|\left\{P\left(f \chi_{j}^{m}\right)(\lambda)\right\}\right\|_{\mathfrak{B}^{s}} \\
& =\sum_{m=1}^{\infty} m^{s}\left(\sum_{j=1}^{2^{m}}\left|P\left(f \chi_{j}^{m}\right)(\lambda)\right|\right)^{1 / 2}
\end{aligned}
$$

Remark 2.3. In the case that $P$ has an integral kernel $p(x, \lambda), G_{P(f)(\lambda)}^{(s)}=\|p(x, \lambda) f(x)\|_{\mathfrak{B}^{s} \text {, }}$ that is the norm of $\left\{\int_{E_{j}^{m}} p(x, \lambda) g(x) d x\right\}$ in $\mathfrak{B}^{s}$.

The following statement is from [4]. We include a proof here for completeness.

Theorem 2.4. [4, Prop.3.3] Given a function $f \in \ell^{p}\left(L^{1}\right)$, fix a martingale structure to $f$. Suppose $P$ is a bounded linear or sublinear operator from $\ell^{p}\left(L^{1}\right)$ to $L^{q}(J)$, where $1 \leq p<2<q$ and $J \subset \mathbb{R}$ is a closed interval. Then

$$
\left\|G_{P(f)(\lambda)}^{(s)}\right\|_{L^{q}(J)} \leq C(p, q, s,\|P\|)\|f\|_{\ell^{p}\left(L^{1}\right)}
$$

Proof. Let

$$
t_{m}(\lambda)=\left(\sum_{j=1}^{2^{m}}\left|P\left(f \chi_{j}^{m}\right)(\lambda)\right|\right)^{1 / 2}
$$

By the definition,

$$
\begin{aligned}
G_{P(f)(\lambda)}^{(s)} & =\sum_{m=1}^{\infty} m^{s}\left(\sum_{j=1}^{2^{m}}\left|P\left(f \chi_{j}^{m}\right)(\lambda)\right|\right)^{1 / 2} \\
& =\sum_{m=1}^{\infty} m^{s} t_{m}(\lambda)
\end{aligned}
$$

Let us give an inequality, for $\gamma \geq 1$

$$
\left(\sum_{i=1}^{N} a_{i}\right)^{\gamma} \leq N^{\gamma-1} \sum_{i=1}^{N}\left|a_{i}\right|^{\gamma} .
$$


Direct computations imply

$$
\begin{aligned}
\int_{J} t_{m}^{q}(\lambda) d \lambda & =\int_{J}\left(\sum_{j=1}^{2^{m}}\left|P\left(f \chi_{j}^{m}\right)(\lambda)\right|^{2}\right)^{q / 2} d \lambda \\
& \leq 2^{m(q / 2-1)} \int_{J} \sum_{j=1}^{2^{m}}\left|P\left(f \chi_{j}^{m}\right)(\lambda)\right|^{q} d \lambda \\
& \stackrel{(15)}{\leq} \quad C 2^{m(q / 2-1)} \sum_{j=1}^{2^{m}}\left\|f \chi_{j}^{m}\right\|_{\ell^{p}\left(L^{1}\right)}^{q} \\
& \stackrel{\text { by (13) }}{\leq} C 2^{m(q / 2-1)} \sum_{j=1}^{2^{m}} 2^{-m \frac{q}{p}}\|f\|_{\ell^{p}\left(L^{1}\right)}^{q} \\
& C \quad\|f\|_{\ell^{p}\left(L^{1}\right)}^{q} 2^{m \frac{q}{2}-m \frac{q}{p}},
\end{aligned}
$$

where the $\star$ holds by the boundedness of $P$.

Finally, we have

$$
\begin{aligned}
\left\|G_{S(f)(\lambda)}^{(s)}\right\|_{L^{q}(J)} & \stackrel{\text { by (14) }}{=}\left\|\sum_{m=1}^{\infty} m^{s} t_{m}(\lambda)\right\|_{L^{q}(J)} \\
& \leq \quad \sum_{m=1}^{\infty} m^{s}\left\|t_{m}(\lambda)\right\|_{L^{q}(J)} \\
& \leq C \sum_{m=1}^{\infty} m^{s} 2^{m / 2-m / p}\|f\|_{\ell^{p}\left(L^{1}\right)} \\
& \leq C\|f\|_{\ell^{p}\left(L^{1}\right)} .
\end{aligned}
$$

Denote by

$$
B_{n}\left(g_{1}, g_{2}, \cdots, g_{n}\right)(x)=\int_{x}^{\infty} \int_{t_{1}}^{\infty} \cdots \int_{t_{n-1}}^{\infty} \prod_{j=1}^{n} g_{j}\left(t_{j}\right) d t_{1} d t_{2} \cdots d t_{n} .
$$

If there is a single function $g$ such that $g_{k}=\{g, \bar{g}\}, k=1,2, \cdots, n$, we write it down by $B_{n}(g)(x)$.

Theorem 2.5. Assume $g_{j}$ is locally integrable, $j=1,2, \cdots, n$. Suppose for $j=$ $1,2, \cdots, n$,

$$
\limsup _{M \rightarrow \infty}\left\|g_{j} \chi_{[M, \infty)}\right\|_{\mathfrak{B}}=0,
$$

and there is a constant $C$ (does not depend on I) such that for any closed interval $I \subset \mathbb{R}_{+}$,

$$
\left\|g_{j} \chi_{I}\right\|_{\mathfrak{B}} \leq C
$$


Then (17) is well defined as the limits

$$
B_{n}\left(g_{1}, g_{2}, \cdots, g_{n}\right)(x)=\lim _{y_{1}, \cdots, y_{n} \rightarrow \infty} \int_{x}^{y_{1}} \int_{t_{1}}^{y_{2}} \cdots \int_{t_{n-1}}^{y_{n}} \prod_{j=1}^{n} g_{j}\left(t_{j}\right) d t_{1} d t_{2} \cdots d t_{n},
$$

and

$$
\lim _{x \rightarrow \infty} B_{n}\left(g_{1}, g_{2}, \cdots, g_{n}\right)(x)=0 .
$$

Moreover, for almost every $x$

$$
\frac{d B_{n}\left(g_{1}, g_{2}, \cdots, g_{n}\right)(x)}{d x}=-g_{1}(x) B_{n-1}\left(g_{2}, \cdots, g_{n}\right)(x) .
$$

Proof. Without loss of generality, assume $n=2$. In order to prove the existence of the limits, it suffices to show that

$$
\lim _{y_{1}, z_{1}, y_{2}, z_{2} \rightarrow \infty} \sup _{x}\left|B_{2}\left(g_{1} \chi_{\left[0, y_{1}\right]}, g_{2} \chi_{\left[0, y_{2}\right]}\right)(x)-B_{2}\left(g_{1} \chi_{\left[0, z_{1}\right]}, g_{2} \chi_{\left[0, z_{2}\right]}\right)(x)\right|=0 .
$$

Assume $M<y_{1}<z_{1}$ and $M<y_{2}<z_{2}$. By telescoping techniques,

$$
\begin{aligned}
& \left|B_{2}\left(g_{1} \chi_{\left[0, y_{1}\right]}, g_{2} \chi_{\left[0, y_{2}\right]}\right)(x)-B_{2}\left(g_{1} \chi_{\left[0, z_{1}\right]}, g_{2} \chi_{\left[0, z_{2}\right]}\right)(x)\right| \\
& \leq \quad\left|B_{2}\left(g_{1} \chi_{\left[0, y_{1}\right]}, g_{2} \chi_{\left[y_{2}, z_{2}\right]}\right)(x)\right|+\left|B_{2}\left(g_{1} \chi_{\left[y_{1}, z_{1}\right]}, g_{2} \chi_{\left[0, z_{2}\right]}\right)(x)\right| \\
& \text { by (11) and [7] } C\left\|g_{1} \chi_{\left[0, y_{1}\right]}\right\|_{\mathfrak{B}}\left\|g_{2} \chi_{\left[y_{2}, z_{2}\right]}\right\|_{\mathfrak{B}}+C\left\|g_{1} \chi_{\left[y_{1}, z_{1}\right]}\right\|_{\mathfrak{B}}\left\|g_{2} \chi_{\left[0, z_{2}\right]}\right\|_{\mathfrak{B}} \\
& \stackrel{\text { by }}{\leq} \quad C\left\|g_{2} \chi_{\left[y_{2}, z_{2}\right]}\right\|_{\mathfrak{B}}+C\left\|g_{1} \chi_{\left[y_{1}, z_{1}\right]}\right\|_{\mathfrak{B}} \\
& \leq \quad C|| g_{2} \chi_{[M, \infty)}\left\|_{\mathfrak{B}}+C\right\| g_{1} \chi_{[M, \infty)} \|_{\mathfrak{B}} .
\end{aligned}
$$

Now (23) follows from (18).

By (20), one has

$$
\begin{aligned}
\lim _{x \rightarrow \infty}\left|B_{2}\left(g_{1}, g_{2}\right)(x)\right| & =\lim _{x \rightarrow \infty} \lim _{x^{\prime} \rightarrow \infty}\left|\int_{x}^{x^{\prime}} \int_{t_{1}}^{x^{\prime}} \prod_{j=1}^{2} g_{j}\left(t_{j}\right) d t_{1} d t_{2}\right| \\
& =\lim _{x \rightarrow \infty} \lim _{x^{\prime} \rightarrow \infty}\left|\int_{x}^{x^{\prime}} \int_{t_{1}}^{x^{\prime}} \prod_{j=1}^{2} g_{j}\left(t_{j}\right) \chi_{[x, \infty)}\left(t_{j}\right) d t_{1} d t_{2}\right| \\
\text { by [11) } & C \lim _{x \rightarrow \infty}\left\|g_{1} \chi_{[x, \infty)}||_{\mathfrak{B}}\right\| g_{2} \chi_{[x, \infty)} \|_{\mathfrak{B}} \\
\text { by [18] } & 0 .
\end{aligned}
$$

This completes the proof of (21). Direct computations imply

$$
\begin{aligned}
\lim _{y \rightarrow x-} \frac{B_{2}\left(g_{1}, g_{2}\right)(y)-B_{2}\left(g_{1}, g_{2}\right)(x)}{y-x} & =\lim _{y \rightarrow x-} \frac{1}{y-x} \int_{y}^{x} g_{1}\left(t_{1}\right) d t_{1} \int_{t_{1}}^{\infty} g_{2}\left(t_{2}\right) d t_{2} \\
& =-g_{1}(x) \int_{x}^{\infty} g_{2}\left(t_{2}\right) d t_{2} .
\end{aligned}
$$


Similarly,

$$
\lim _{y \rightarrow x+} \frac{B_{2}\left(g_{1}, g_{2}\right)(y)-B_{2}\left(g_{1}, g_{2}\right)(x)}{y-x}=-g_{1}(x) \int_{x}^{\infty} g_{2}\left(t_{2}\right) d t_{2} .
$$

The last two equalities imply (22).

Remark 2.6. In [4, Prop.4.1], a weaker limit $\tilde{B}_{n}\left(g_{1}, g_{2}, \cdots, g_{n}\right)(x)$ was proved to be existed, where

$$
\tilde{B}_{n}\left(g_{1}, g_{2}, \cdots, g_{n}\right)(x)=\lim _{y \rightarrow \infty} \int_{x}^{y} \int_{t_{1}}^{y} \cdots \int_{t_{n-1}}^{y} \prod_{j=1}^{n} g_{j}\left(t_{j}\right) d t_{1} d t_{2} \cdots d t_{n} .
$$

Let $p(x, \lambda)$ be a measurable function on $\mathbb{R}_{+} \times J$. Define the integral operator $P$ :

$$
P(f)(\lambda)=\int_{\mathbb{R}_{+}} p(x, \lambda) f(x) d x
$$

and the maximal operator $P^{*}$ :

$$
P^{*}(f)(\lambda)=\sup _{y \in \mathbb{R}_{+}}\left|\int_{y}^{\infty} p(x, \lambda) f(x) d x\right| .
$$

Lemma 2.7. [2, Christ-Kiselev lemma] Let $1 \leq p<q<\infty$. Suppose $P$ is a bounded operator from $\ell^{p}\left(L^{1}\right)$ to $L^{q}(J)$. Then $P^{*}$ is also a bounded operator from $\ell^{p}\left(L^{1}\right)$ to $L^{q}(J)$.

In our situation (see next section), $s(x, \lambda)=w(x, \lambda) e^{-i h(x, \lambda)}$, where $h$ is a realvalued function. We obtain two corresponding operators

$$
S(f)(\lambda)=\int_{\mathbb{R}_{+}} w(x, \lambda) e^{-i h(x, \lambda)} f(x) d x
$$

and

$$
S^{*}(f)(\lambda)=\sup _{y \in \mathbb{R}_{+}}\left|\int_{y}^{\infty} w(x, \lambda) e^{-i h(x, \lambda)} f(x) d x\right| .
$$

Lemma 2.8. Assume $1 \leq p \leq 2$. Suppose there exist a constant $C$ and a open interval $\tilde{J}$ satisfying $J \subset \tilde{J}$ such that for $\lambda \in \tilde{J}$

$$
\left|\partial_{\lambda}[h(x, \lambda)-h(y, \lambda)]\right| \geq \frac{|x-y|}{C}
$$

and for $i=1,2,3$ and $\lambda \in \tilde{J}$

$$
\left|\partial_{\lambda}^{i}[h(x, \lambda)-h(y, \lambda)]\right| \leq C|x-y|
$$

provided $|x-y| \geq C$. Suppose

$$
\sup _{x \in \mathbb{R}_{+}, \lambda \in \tilde{J}} \sum_{i=1}^{2}\left|\partial_{\lambda}^{i} w(x, \lambda)\right| \leq C .
$$

Let $p^{\prime}=\frac{p}{p-1}$ be the conjugate exponent to $p$. Then

$$
\|S f\|_{L^{p^{\prime}}(J, d \lambda)} \leq O(1)\|f\|_{\ell^{p}\left(L^{1}\right)},
$$


and

$$
\left\|S^{*} f\right\|_{L^{p^{\prime}(J, d \lambda)}} \leq O(1)\|f\|_{\ell^{p}\left(L^{1}\right)},
$$

where $O(1)$ depends on $C, J, \tilde{J}$ and $p$.

Proof. By Lemma 2.7, we only need to prove the boundedness of $S$. By interpolation theorem, it suffices to prove the cases $p=1$ and $p=2$. The case of $p=1$ is trivial since $h$ is a real-valued function. So we only need to consider the case $p=2$. Let $\xi(\lambda)$ be a positive function so that $\xi \equiv 1$ on $J$ and $\operatorname{supp} \xi \subset \tilde{J}$. Then one has

$$
\begin{aligned}
\|S f\|_{L^{2}(J, d \lambda)}^{2} & =\int_{J}\left|\int_{\mathbb{R}_{+}} w(x, \lambda) e^{-i h(x, \lambda)} f(x) d x\right|^{2} d \lambda \\
& \leq \int_{\tilde{J}}\left|\int_{\mathbb{R}_{+}} w(x, \lambda) e^{-i h(x, \lambda)} f(x) d x\right|^{2} \xi(\lambda) d \lambda \\
& =\int_{\tilde{J}}\left[\int_{\mathbb{R}_{+}} w(x, \lambda) e^{-i h(x, \lambda)} f(x) d x\right]\left[\int_{\mathbb{R}_{+}} \bar{w}(y, \lambda) e^{i h(y, \lambda)} \bar{f}(y) d y\right] \xi(\lambda) d \lambda \\
& =\int_{\mathbb{R}_{+}^{2}} f(x) \bar{f}(y) d x d y \int_{\tilde{J}} e^{-i h(x, \lambda)+i h(y, \lambda)} w(x, \lambda) \bar{w}(y, \lambda) \xi(\lambda) d \lambda .
\end{aligned}
$$

Multiplying $-i \partial_{\lambda}(h(x, \lambda)-h(y, \lambda))$ and dividing $-i \partial_{\lambda}(h(x, \lambda)-h(y, \lambda))$ and integrating by part twice, we have for $|x-y| \geq C$,

$$
\begin{aligned}
& \int_{\tilde{J}} e^{-i h(x, \lambda)+i h(y, \lambda)} w(x, \lambda) \bar{w}(y, \lambda) \xi(\lambda) d \lambda \\
& =\int_{\tilde{J}} \frac{-i \partial_{\lambda}(h(x, \lambda)-h(y, \lambda))}{-i \partial_{\lambda}(h(x, \lambda)-h(y, \lambda))} e^{-i h(x, \lambda)+i h(y, \lambda)} w(x, \lambda) \bar{w}(y, \lambda) \xi(\lambda) d \lambda \\
& =\int_{\tilde{J}} e^{-i h(x, \lambda)+i h(y, \lambda)} \partial_{\lambda}\left(\frac{w(x, \lambda) \bar{w}(y, \lambda) \xi(\lambda)}{-i \partial_{\lambda}(h(x, \lambda)-h(y, \lambda))}\right) d \lambda \\
& =\int_{\tilde{J}} e^{-i h(x, \lambda)+i h(y, \lambda)} \partial_{\lambda}\left[\frac{1}{-i \partial_{\lambda}(h(x, \lambda)-h(y, \lambda))} \partial_{\lambda}\left(\frac{w(x, \lambda) \bar{w}(y, \lambda) \xi(\lambda)}{-i \partial_{\lambda}(h(x, \lambda)-h(y, \lambda))}\right)\right] d \lambda \\
& =\frac{O(1)}{|x-y|^{2}}
\end{aligned}
$$

where the last equality holds by Lemma 4.1 .

By (29) and (30), we have

$$
\begin{aligned}
\|S f\|_{L^{2}(J, d \lambda)}^{2} \leq & \int_{|x-y|>C} f(x) \bar{f}(y) d x d y \int_{\tilde{J}} e^{-i h(x, \lambda)+i h(y, \lambda)} w(x, \lambda) \bar{w}(y, \lambda) \xi(\lambda) d \lambda . \\
& +\int_{|x-y| \leq C} f(x) \bar{f}(y) d x d y \int_{\tilde{J}} e^{-i h(x, \lambda)+i h(y, \lambda)} w(x, \lambda) \bar{w}(y, \lambda) \xi(\lambda) d \lambda \\
= & O(1) \int_{\mathbb{R}_{+}^{2}} \frac{|f(x) f(y)|}{1+|x-y|^{2}} d x d y \\
= & O(1)\|f\|_{\ell^{2}\left(L^{1}\right)},
\end{aligned}
$$


where the the last equality holds by calculations (for convenience, we include the details in the appendix). This completes the proof.

Remark 2.9. The formulation and proof of Lemma 2.8 closely follows from the corresponding parts appearing in [3, 4, 12].

\section{Preparations for the applications}

In the rest of this paper, we will apply Theorem 2.5 to prove Theorems 1.1, 1.2 and 1.5. Let us set up the basics in this section and do the proof in the following sections. Since $\varphi(x, E)$ is the Floquet solution for $E \in \cup\left(a_{n}, b_{n}\right)$, one has

$$
\varphi(x, E)=J(x, E) e^{i k(E) x},
$$

where $k(E) \in[0, \pi]$ is the quasimomentum, and $J(x, E)$ is 1-periodic. Since $\varphi(x, E)$ and $\bar{\varphi}(x, E)$ are two linearly independent solutions of $u^{\prime \prime}+V_{0} u=E u$, the Wronskian $W(\bar{\varphi}, \varphi)$ is constant and

$$
W(\bar{\varphi}, \varphi)=\bar{\varphi}(x) \varphi^{\prime}(x)-\bar{\varphi}^{\prime}(x) \varphi(x)=2 i \Im\left[\bar{\varphi}(x) \varphi^{\prime}(x)\right] .
$$

By interchanging $\bar{\varphi}$ and $\varphi$, we always assume

$$
W(\bar{\varphi}, \varphi)=i \omega
$$

with $\omega>0$.

Let us study the solutions of the equation

$$
-u^{\prime \prime}+\left(V_{0}(x)+V(x)\right) u=E u .
$$

We rewrite this equation as a linear system

$$
u_{1}^{\prime}=\left(\begin{array}{cc}
0 & 1 \\
V_{0}+V-E & 0
\end{array}\right) u_{1},
$$

where $u_{1}$ is a vector $\left(\begin{array}{c}u \\ u^{\prime}\end{array}\right)$. Introduce

$$
u_{1}=\left(\begin{array}{cc}
\varphi(x, E) & \bar{\varphi}(x, E) \\
\varphi^{\prime}(x, E) & \bar{\varphi}^{\prime}(x, E)
\end{array}\right) u_{2} .
$$

Then

$$
u_{2}^{\prime}=\frac{i}{2 \Im\left(\varphi \bar{\varphi}^{\prime}\right)}\left(\begin{array}{cc}
V(x)|\varphi(x, E)|^{2} & V(x) \bar{\varphi}(x, E)^{2} \\
-V(x) \varphi(x, E)^{2} & -V(x)|\varphi(x, E)|^{2}
\end{array}\right) u_{2} .
$$

Define

$$
p(x, E)=\frac{1}{2 \Im\left(\varphi \overline{\varphi^{\prime}}\right)} \int_{0}^{x} V(y)|\varphi(y, E)|^{2} d y .
$$

Let us apply another transformation,

$$
u_{2}=\left(\begin{array}{cc}
\exp (i p(x, E)) & 0 \\
0 & \exp (-i p(x, E))
\end{array}\right) u_{3} .
$$


We obtain the equation for $u_{3}$ :

(36)

$$
u_{3}^{\prime}=\frac{i}{2 \Im\left(\varphi \bar{\varphi}^{\prime}\right)}\left(\begin{array}{cc}
0 & V(x) \bar{\varphi}(x, E)^{2} \exp (-2 i p(x, E)) \\
-V(x) \varphi(x, E)^{2} \exp (2 i p(x, E)) & 0
\end{array}\right) u_{3} .
$$

Lemma 3.1. Suppose there exists a solution of (36) satisfying

$$
u_{3}(x, E)=\left(\begin{array}{l}
1 \\
0
\end{array}\right)+o(x)
$$

as $x \rightarrow \infty$. Then there exists a solution $u(x, E)$ of (1) satisfying (4).

Proof. The proof is straitfoward by subistitutions.

Let $Y=u_{3}$. Let $\phi$ be so that $e^{i \phi}=\varphi(x, E)$. Denote by

$$
w(x, E)=\frac{i}{2 \Re \phi^{\prime}},
$$

and

$$
h(x, E)=2 \Re \phi-\int_{0}^{x} \frac{V(t)}{\Re \phi^{\prime}(t, E)} d t .
$$

In the following, $w$ and $h$ are always given by (37) and (38) correspondingly. The operators $S$ and $S^{*}$ are given by (25) and (26) correspondingly. Denote by

$$
\mathcal{F}(x, E)=w(x, E) e^{-i h(x, E)} V(x) .
$$

Under those notations and following calcaluations in p. 249 and p.250 in [3], (36]) becomes

$$
Y^{\prime}=\left(\begin{array}{cc}
0 & w e^{-i h} V \\
\bar{w} e^{i h} V & 0
\end{array}\right) Y=\left(\begin{array}{cc}
0 & \mathcal{F} \\
\overline{\mathcal{F}} & 0
\end{array}\right) Y
$$

For convenience, we include a verification of (40) in the Appendix.

Denote by

$$
D=\left(\begin{array}{cc}
0 & \mathcal{F} \\
\overline{\mathcal{F}} & 0
\end{array}\right)
$$

The linear equation (40) becomes $Y^{\prime}=D Y$. We are going to find a solution as

$$
Y(x)=\left(\begin{array}{l}
1 \\
0
\end{array}\right)-\int_{x}^{\infty} D(y) Y(y) d y
$$

and we obtain a series solution by iterations

$Y(x)=\left(\begin{array}{l}1 \\ 0\end{array}\right)+\sum_{k=1}^{\infty}(-1)^{k} \int \cdots \int_{x \leq t_{1} \leq t_{2} \cdots \leq t_{k}<\infty} D\left(t_{1}\right) D\left(t_{2}\right) \cdots D\left(t_{k}\right)\left(\begin{array}{l}1 \\ 0\end{array}\right) d t_{k} d t_{k-1} \cdots d t_{2} d t_{1}$.

Let

$$
T_{n}(\mathcal{F})\left(x, x^{\prime}, E\right)=M_{n}(\mathcal{F}(\cdot, E))\left(x, x^{\prime}\right) .
$$

Under those notations, one has

$$
\int \cdots \int_{x \leq t_{1} \leq t_{2} \cdots \leq t_{2 k} \leq x^{\prime}} D\left(t_{1}\right) D\left(t_{2}\right) \cdots D\left(t_{2 k}\right)\left(\begin{array}{l}
1 \\
0
\end{array}\right) d t_{2 k} \cdots d t_{2} d t_{1}=\left(\begin{array}{c}
T_{2 k}(\mathcal{F})\left(x, x^{\prime}, E\right) \\
0
\end{array}\right),
$$


and

$$
\int \cdots \int_{x \leq t_{1} \leq t_{2} \cdots \leq t_{2 k+1} \leq x^{\prime}} D\left(t_{1}\right) D\left(t_{2}\right) \cdots D\left(t_{2 k+1}\right)\left(\begin{array}{l}
1 \\
0
\end{array}\right) d t_{2 k+1} \cdots d t_{2} d t_{1}=\left(\begin{array}{c}
0 \\
T_{2 k+1}(\mathcal{F})\left(x, x^{\prime}, E\right)
\end{array}\right) .
$$

The series solution (42) becomes

$$
Y(x)=\left(\begin{array}{l}
1 \\
0
\end{array}\right)+\left(\begin{array}{c}
\sum_{m=1}^{\infty} T_{2 m}(\mathcal{F})(x, \infty, E) \\
-\sum_{m=0}^{\infty} T_{2 m+1}(\mathcal{F})(x, \infty, E)
\end{array}\right) .
$$

We will show (42) and (43) are well defined and give an actual solution of (40).

\section{Proof of Theorem 1.1}

Fix a martingale structure $\left\{E_{j}^{m} \subset \mathbb{R}_{+}: m \in \mathbb{Z}_{+}, 1 \leq j \leq 2^{m}\right\}$ to the potential $V$. Choose a spectral band $\left(a_{n}, b_{n}\right)$ and let $K \subset\left(a_{n}, b_{n}\right)$ be an arbitrary closed interval. We will apply Theorem 2.5 to complete our proof. In order to make it different from the abstract statements, instead of using parameter $\lambda$, the energy parameter $E$ will be used.

Lemma 4.1. For any $E \in\left(a_{n}, b_{n}\right)$, there exists a constant $C=C(E)$ (depends on $E$ uniformly in any compact subset of $\left(a_{n}, b_{n}\right)$ ) such that

$$
\left|\partial_{E}[h(x, E)-h(y, E)]\right| \geq \frac{|x-y|}{C}
$$

and for $i=1,2,3$

$$
\left|\partial_{E}^{i}[h(x, E)-h(y, E)]\right| \leq C|x-y|
$$

provided $|x-y| \geq C$.

Proof. We will prove (44) first. By the definition of $\phi$ and (32), one has

$$
\Re \phi=k(E) x+\Im \log J(x, E),
$$

with $k(E) \in(0, \pi)$. By the Floquet theory,

$$
\frac{d k(E)}{d E} \neq 0
$$

By the fact that $J(x, E)$ is 1-periodic, one has

$$
\Im \log J(x+1, E)-\Im \log J(x, E)=2 q \pi,
$$

for some $q \in \mathbb{Z}$. It implies

$$
\partial_{E}(\Im \log J(x+1, E)-\Im \log J(x, E))=0 .
$$

By (47) and (49), we have

$$
|\Re \phi(x, E)-\Re \phi(y, E)| \geq \frac{|x-y|}{C} .
$$

Since $V(x) \in \ell^{p}\left(L^{1}\right)$, one has $\partial_{E} \int_{N}^{N+1} \frac{V(t)}{\Re \phi^{\prime}(t, E)} d t$ goes to zero as $N \rightarrow \infty$. It implies

$$
\left|\partial_{E} \int_{x}^{y} \frac{V(t)}{\Re \phi^{\prime}(t, E)} d t\right|=o(y-x)+O(1),
$$


as $y-x$ goes to $\infty$. Now (44) follows from (50) and (51). The proof of (45) can be proceeded in a similar way.

Lemma 4.2. Let $q$ be the number conjugate to $p$ with $1 \leq p<2$. Then the following estimate holds

In particular $(s=1)$,

$$
G_{S^{*}(V)(E)}^{(s)} \in L^{q}(K, d E) .
$$

$$
G_{S^{*}(V)(E)} \in L^{q}(K, d E) .
$$

Proof. By Lemmas 4.1, 2.8 and applying $P=S^{*}$ in Theorem 2.4, we have

$$
G_{S^{*}(V)(E)}^{(s)} \in L^{q}(K, d E) .
$$

Corollary 4.3. Let $q$ be the number conjugate to $p$ with $1 \leq p<2$. Then for almost every $E \in K$,

$$
\left\|\mathcal{F}(\cdot, E) \chi_{I}\right\|_{\mathfrak{B}} \leq C(E),
$$

for any closed interval $I$, and

$$
\limsup _{M \rightarrow \infty}\left\|\mathcal{F}(\cdot, E) \chi_{[M, \infty)}\right\|_{\mathfrak{B}}=0 .
$$

Proof. Direct computations, one has

$$
\begin{aligned}
\left\|\mathcal{F}(\cdot, E) \chi_{I}\right\|_{\mathfrak{B}} & =\left\|\left\{\int_{E_{j}^{m}} \mathcal{F}(x, E) \chi_{I}(x) d x\right\}\right\|_{\mathfrak{B}} \\
& =\left\|\left\{\int_{E_{j}^{m}} w(x, E) e^{i h(x, E)} V(x) \chi_{I}(x) d x\right\}\right\|_{\mathfrak{B}} \\
& =\left\|\left\{\int_{I} w(x, E) e^{i h(x, E)} V(x) \chi_{j}^{m} d x\right\}\right\|_{\mathfrak{B}} \\
& \leq 2\left\|\left\{S^{*}\left(V \chi_{j}^{m}\right)(E)\right\}\right\|_{\mathfrak{B}} \\
& =2 G_{S^{*}(V)(E) .}
\end{aligned}
$$

Now (53) follows since $G_{S^{*}(V)(E)} \in L^{q}(K, d E)$ by Lemma 4.2. Applying $P=S$ and $f=V(x) \chi_{[M, \infty)}$ in Theorem 2.4 and recalling that

$$
\left\|\mathcal{F}(\cdot, E) \chi_{[M, \infty)}\right\|_{\mathfrak{B}}=G_{S\left(V \chi_{[M, \infty)}\right)},
$$

one has

$$
\begin{aligned}
\limsup _{M \rightarrow \infty}\left(\int_{J}\left\|\mathcal{F}(\cdot, E) \chi_{[M, \infty)}\right\|_{\mathfrak{B}}^{q} d E\right)^{1 / q} & =\limsup _{M \rightarrow \infty}\left\|G_{S\left(V \chi_{[M, \infty)}\right)}\right\|_{L^{q}(J)} \\
& \leq O(1) \limsup _{M \rightarrow \infty}\left\|V \chi_{[M, \infty)}\right\|_{\ell^{p}\left(L^{1}\right)} \\
& =0 .
\end{aligned}
$$

It implies for almost every $E \in K$,

$$
\limsup _{M \rightarrow \infty}\left\|\mathcal{F}(\cdot, E) \chi_{[M, \infty)}\right\|_{\mathfrak{B}}=0 .
$$


This yields to (54).

Proof of Theorem 1.1. Under the assumption of Theorem 1.1, $\sigma_{\text {ess }}(H)=\sigma_{\text {ess }}\left(H_{0}\right)$ [21, 40]. This yields that $\sigma_{a c}(H) \subset S$. It is well known that boundedness of the eigensolution implies purely absolutely continuous spectrum (e.g. [36, 41]). Thus the second part of Theorem 1.1 implies the first part. If $p=1\left(V \in L^{1}\left(\mathbb{R}^{+}\right)\right)$, one has that for every $E \in K, \frac{i V}{2 \Re \phi^{\prime}} e^{i h}$ given by (40) is in $L^{1}$. In this case, it is well known (see [7] for example) that (40) has a solution $Y(x)$ satisfying

$$
Y(x)=\left(\begin{array}{l}
1 \\
0
\end{array}\right)+o(1)
$$

as $x \rightarrow \infty$. By Lemma (3.1), Theorem 1.1 is true for $p=1$. So we assume $1<p<2$.

By Corollary 4.3 and Theorem 2.5, for almost every $E \in K$ the following limit is well defined,

$$
T_{2 m}(\mathcal{F})(x, \infty, E)=\lim _{x^{\prime} \rightarrow \infty} T_{2 m}(\mathcal{F})\left(x, x^{\prime}, E\right) .
$$

By (12) and (53), we have

$$
\left|T_{2 m}(\mathcal{F})(x, \infty, E)\right| \leq \frac{C(E)^{2 m}}{\sqrt{(2 m) !}} .
$$

Thus

$$
\sum_{m=1}^{\infty} T_{2 m}(\mathcal{F})(x, \infty, E)
$$

is absolutely convergent for almost every $E \in K$. Similarly,

$$
\sum_{m=0}^{\infty} T_{2 m+1}(\mathcal{F})(x, \infty, E)
$$

is absolutely convergent for almost every $E \in K$.

(57) and (58) show that the right hand series in (42) and (43) are well defined for almost every $E \in K$. Based on (22), it is easy to check for almost every $E \in K$, the right hand series in (42) and (43) actually gives a solution of (40). The WKB behavior (44) follows by (21) and Lemma 3.1 .

\section{An alternative proof of Theorem 1.1 and proof of Theorem 1.2}

We will give a new proof of Theorem 1.1. As the arguments in the previous section, it suffices to prove Corollary 4.3. The proof of Corollary 4.3 in the previous section is based on the maximal operator $S^{*}$. We will give a new proof without using the maximal operator.

Lemma 5.1. [2] Let $\left\{E_{j}^{m} \subset \mathbb{R}_{+}: m \in \mathbb{Z}_{+}, 1 \leq j \leq 2^{m}\right\}$ be a martingale structure. Then there exists an absolute constant $C$ such that for any closed interval $I$

$$
\left\|g \chi_{I}\right\|_{\mathfrak{B}^{s}} \leq C\|g\|_{\mathfrak{B}^{s+1}} .
$$

In particular,

$$
\left\|g \chi_{I}\right\|_{\mathfrak{B}} \leq C\|g\|_{\mathfrak{B}^{2}}
$$


A new proof of Corollary 4.3 without using $S^{*}$. The proof of (54) does not use $S^{*}$. So we keep it. We only need to show (53) is true for almost every $E \in K$. Applying $P=S$ and $f=V(x)$ in Theorem 2.4 and recalling that

$$
\|\mathcal{F}(\cdot, E)\|_{\mathfrak{B}^{2}}=G_{S(V)(E)}^{(2)},
$$

one has

$$
\|\mathcal{F}(\cdot, E)\|_{\mathfrak{B}^{2}} \in L^{q}(J) .
$$

Now (53) follows from (59) and (60).

Suppose the assumptions of Theorem 1.2 hold for some $1<p \leq 2$ and $\gamma>0$. Let $\beta$ be any positive number bigger than $1-p^{\prime} \gamma$. Denote by $\mathcal{H}^{\beta}$ the $\beta$-dimensional Hausdorff measure. Let

$$
\Lambda_{c}=\left\{E \in K:\left\|\mathcal{F}(\cdot, E) \chi_{[N, \infty)}\right\|_{\mathfrak{B}^{2}} \geq c \text { for every } N \geq 0\right\} .
$$

Lemma 5.2. For any $c>0$, we have

$$
\mathcal{H}^{\beta}\left(\Lambda_{c}\right)=0 .
$$

Proof. The Lemma follows from the arguments in [3]. Actually, the proof of Lemma 5.2 is easier since the potential $V$ does not depend on $E$.

Proof of Theorem 1.2. By Lemma 5.2, we have for all $E$ except for a set of $\mathcal{H}^{\beta}$ zero, for any $c>0$, there exists $N>0$ such that

$$
\left\|\mathcal{F}(\cdot, E) \chi_{[N, \infty)}\right\|_{\mathfrak{B}^{2}} \leq c .
$$

Fix such $E$. Let $N_{0}$ be such that (61) holds for $c=1$. By changing $x$ to $x-N_{0}$, we can assume $N_{0}=0$. Hence, by (59), one has

$$
\sup _{I \subset \mathbb{R}_{+}}\left\|\mathcal{F}(\cdot, E) \chi_{I}\right\|_{\mathfrak{B}} \leq C .
$$

For any $\varepsilon>0$, let $N(\varepsilon)$ be large enough so that (61) holds for $c=\varepsilon$. For any $M>N(\varepsilon)$, by (59) again,

$$
\begin{aligned}
\left\|\mathcal{F}(\cdot, E) \chi_{[M, \infty)}\right\|_{\mathfrak{B}} & \leq\left\|\mathcal{F}(\cdot, E) \chi_{[N(\varepsilon), \infty)}\right\|_{\mathfrak{B}}+\left\|\mathcal{F}(\cdot, E) \chi_{[N(\varepsilon), M)}\right\|_{\mathfrak{B}} \\
& \leq\left\|\mathcal{F}(\cdot, E) \chi_{[N(\varepsilon), \infty)}\right\|_{\mathfrak{B}}+C\left\|\mathcal{F}(\cdot, E) \chi_{[N(\varepsilon), \infty)}\right\|_{\mathfrak{B}^{2}} \\
& \leq C \varepsilon
\end{aligned}
$$

This implies (54). Now the rest proof of Theorem 1.2 follows from that of Theorem 1.1

\section{Sharp estimates For almost orthogonality among Generalized PRÜFER ANGLES}

In this section, we always assume for some $B>0$,

$$
|V(x)| \leq \frac{B}{1+x} .
$$

Without loss generality, we only consider the Dirichlet boundary condition. 
For any spectral band $\left[a_{n}, b_{n}\right]$, let $c_{n}$ be the unique number such that $k\left(c_{n}\right)=\frac{\pi}{2}$. Let $I$ be a closed interval in $\left(a_{n}, c_{n}\right)$ or $\left(c_{n}, b_{n}\right)$. All the energies $E$ in this section are in $I$ and the estimates are uniform with respect to $E \in I$.

For $z \in \mathbb{C} \backslash \mathbb{R}$, denote by $\tilde{v}(x, z)(\tilde{u}(x, z))$ the solution of $H_{0}+V$ with boundary condition $\tilde{v}(0, z)=1$ and $\tilde{v}^{\prime}(0, z)=0\left(\tilde{u}(0, z)=0\right.$ and $\left.\tilde{u}^{\prime}(0, z)=1\right)$. The Weyl $m$ function $m(z)$ (well defined on $z \in \mathbb{C} \backslash \mathbb{R}$ ) is given by the unique complex number $m(z)$ so that $\tilde{v}(x, z)+m(z) \tilde{u}(x, z) \in L^{2}\left(\mathbb{R}^{+}\right)$. The spectral measure $\mu$ on $\mathbb{R}$, is given by the following formula, for $z \in \mathbb{C} \backslash \mathbb{R}$

$$
m(z)=C+\int\left[\frac{1}{x-z}-\frac{x}{1+x^{2}}\right] d \mu(x),
$$

where $C \in \mathbb{R}$ is a constant.

Denote $\mu_{s c}$ by the singular continuous component of $\mu$. The spectral measure $\mu$ can determine the spectra of $H$. For example, $\sigma_{\mathrm{sc}}\left(H_{0}+V\right)=\emptyset$ if and only if $\mu_{s c}=0$.

Define $\gamma(x, E)$ as a continuous function such that

$$
\varphi(x, E)=|\varphi(x, E)| e^{i \gamma(x, E)} .
$$

By [17, Proposition 2.1], we know there exists some constant $C>0$ such that

$$
\frac{1}{C} \leq \gamma^{\prime}(x, E) \leq C
$$

Define $\rho(x, E) \in \mathbb{C}$ by

$$
\left(\begin{array}{c}
u(x, E) \\
u^{\prime}(x, E)
\end{array}\right)=\frac{1}{2 i}\left[\rho(x, E)\left(\begin{array}{c}
\varphi(x, E) \\
\varphi^{\prime}(x, E)
\end{array}\right)-\bar{\rho}(x, E)\left(\begin{array}{c}
\bar{\varphi}(x, E) \\
\bar{\varphi}^{\prime}(x, E)
\end{array}\right)\right] .
$$

We should mention that $u(x, E)$ is a real function and $\varphi(x, E)$ is not.

Define $R(x, E)$ and $\theta(x, E)$ by

$$
R(x, E)=|\rho(x, E)| ; \quad \theta(x, E)=\gamma(x, E)+\operatorname{Arg}(\rho(x, E)) .
$$

Proposition 6.1. [17] Suppose $u$ is a real solution of (11). Then there exist real functions $R(x)>0$ and $\theta(x)$ such that

$$
[\ln R(x, E)]^{\prime}=\frac{V(x)}{2 \gamma^{\prime}(x, E)} \sin 2 \theta(x, E)
$$

and

$$
\theta(x, E)^{\prime}=\gamma^{\prime}(x, E)-\frac{V(x)}{2 \gamma^{\prime}(x, E)} \sin ^{2} \theta(x, E) .
$$

In the following, we will prove the almost orthogonality among Prüfer angles in a sharp way. Before the proof, some preparations are necessary.

Lemma 6.2. [15] Suppose the function $G(x)$ satisfies $\left|G^{\prime}(x)\right|=\frac{O(1)}{1+x}$ and $\gamma \neq 0$. Then

$$
\left|\int_{0}^{L} \frac{\sin (\gamma x+G(x))}{1+x} d x\right| \leq O(1) \log \left|\gamma^{-1}\right|+O(1) .
$$


Lemma 6.3. 25] Suppose the function $G(x)$ satisfies $\left|G^{\prime}(x)\right|=\frac{O(1)}{1+x}$ and $\gamma \neq 0$. Then

$$
\left|\int_{0}^{L} \frac{\sin (\gamma x+G(x))}{1+x} d x\right| \leq \frac{O(1)}{|\gamma|}+O(1)
$$

Lemma 6.4. Suppose $0<\gamma<2 \pi$ and the function $G(x)$ satisfies $\left|G^{\prime}(x)\right|=\frac{O(1)}{1+x}$. Then

$$
\left|\int_{0}^{L} e^{2 \pi i k x} \frac{\sin (\gamma x+G(x))}{1+x} d x\right| \leq O(1) \log \gamma^{-1}+O(1) \log (2 \pi-\gamma)^{-1}+O(1)
$$

for $k=-1,0,1$, and

$$
\left|\int_{0}^{L} e^{2 \pi i k x} \frac{\sin (\gamma x+G(x))}{1+x} d x\right| \leq \frac{O(1)}{|k|}+O(1)
$$

for $k \in \mathbb{Z} \backslash\{-1,0,1\}$.

Proof. The proof follows from Lemmas 6.2, 6.3 and trigonometric identity.

Denote by $\mathbb{T}=\mathbb{R} / \mathbb{Z}$.

Theorem 6.5. Suppose $f \in L^{2}(\mathbb{T})$. Then the following estimates hold

$$
\left|\int_{0}^{L} f(x) \frac{\cos 4 \theta(x, E)}{1+x} d x\right|=O(1)
$$

and

$$
\left|\int_{0}^{L} f(x) \frac{\sin 2 \theta\left(x, E_{1}\right) \sin 2 \theta\left(x, E_{2}\right)}{1+x} d x\right|=O(1) \log \frac{1}{\left|E_{1}-E_{2}\right|}+O(1),
$$

where $O(1)$ only depends on $I, B, f$ and $V_{0}$.

Proof. We only give the proof of (170). First, using (68) and (62) we have the differential equations of $\theta\left(x, E_{1}\right)$ and $\theta\left(x, E_{2}\right)$,

$$
\theta^{\prime}\left(x, E_{1}\right)=\gamma^{\prime}\left(x, E_{1}\right)+\frac{O(1)}{1+x}
$$

and

$$
\theta^{\prime}\left(x, E_{2}\right)=\gamma^{\prime}\left(x, E_{2}\right)+\frac{O(1)}{1+x} .
$$

By (32) and (63), we have

$$
\gamma(x, E)=k(E) x+\eta(x, E)
$$

where $\eta(x, E) \bmod 2 \pi$ is a function that is 1 -periodic in $x$.

By basic trigonometry,

$-2 \sin 2 \theta\left(x, E_{1}\right) \sin 2 \theta\left(x, E_{2}\right)=\cos \left(2 \theta\left(x, E_{1}\right)+2 \theta\left(x, E_{2}\right)\right)-\cos \left(2 \theta\left(x, E_{1}\right)-2 \theta\left(x, E_{2}\right)\right)$,

it suffices to bound

$$
\int_{0}^{L} f(x) \frac{\cos \left(2 \theta\left(x, E_{1}\right) \pm 2 \theta\left(x, E_{2}\right)\right)}{1+x} d x
$$


Without of loss of generality, we only bound

$$
\int_{0}^{L} f(x) \frac{\cos \left(2 \theta\left(x, E_{1}\right)-\theta\left(x, E_{2}\right)\right)}{1+x} d x .
$$

By (71), (72) and (73), we have

$$
\frac{d}{d x}\left(\left[\theta\left(x, E_{1}\right)-\eta\left(x, E_{1}\right)\right]-\left[\theta\left(x, E_{2}\right)-\eta\left(x, E_{2}\right)\right]=k\left(E_{1}\right)-k\left(E_{2}\right)+\frac{O(1)}{1+x} .\right.
$$

Let

$$
\tilde{\theta}(x, E)=\theta(x, E)-\eta(x, E) .
$$

By trigonometry again, one has

$$
\begin{aligned}
\cos \left(2 \theta\left(x, E_{1}\right)-2 \theta\left(x, E_{2}\right)\right)= & \cos \left(2 \tilde{\theta}\left(x, E_{1}\right)-2 \tilde{\theta}\left(x, E_{2}\right)+2 \eta\left(x, E_{1}\right)-2 \eta\left(x, E_{2}\right)\right) \\
= & \cos \left(2 \eta\left(x, E_{1}\right)-2 \eta\left(x, E_{2}\right)\right) \cos \left(2 \tilde{\theta}\left(x, E_{1}\right)-2 \tilde{\theta}\left(x, E_{2}\right)\right) \\
& -\sin \left(2 \eta\left(x, E_{1}\right)-2 \eta\left(x, E_{2}\right)\right) \sin \left(2 \tilde{\theta}\left(x, E_{1}\right)-2 \tilde{\theta}\left(x, E_{2}\right)\right) .
\end{aligned}
$$

Thus

$$
\begin{aligned}
\int_{0}^{L} f(x) \frac{\cos \left(2 \theta\left(x, E_{1}\right)-2 \theta\left(x, E_{2}\right)\right)}{1+x} d x & \\
= & \int_{0}^{L} f(x) \frac{\cos \left(2 \eta\left(x, E_{1}\right)-2 \eta\left(x, E_{2}\right)\right) \cos \left(2 \tilde{\theta}\left(x, E_{1}\right)-2 \tilde{\theta}\left(x, E_{2}\right)\right)}{1+x} d x \\
& -\int_{0}^{L} f(x) \frac{\sin \left(2 \eta\left(x, E_{1}\right)-2 \eta\left(x, E_{2}\right)\right) \sin \left(2 \tilde{\theta}\left(x, E_{1}\right)-2 \tilde{\theta}\left(x, E_{2}\right)\right)}{1+x} d x .
\end{aligned}
$$

Without loss of generality, we only give the estimate for

$$
\int_{0}^{L} f(x) \frac{\sin \left(2 \eta\left(x, E_{1}\right)-2 \eta\left(x, E_{2}\right)\right) \sin \left(2 \tilde{\theta}\left(x, E_{1}\right)-2 \tilde{\theta}\left(x, E_{2}\right)\right)}{1+x} d x .
$$

We proceed by Fourier expansion of $f(x) \sin \left(2 \eta\left(x, E_{1}\right)-2 \eta\left(x, E_{2}\right)\right)$ (1-periodic function) and obtain that

$$
f(x) \sin \left(2 \eta\left(x, E_{1}\right)-2 \eta\left(x, E_{2}\right)\right)=\frac{c_{0}}{2}+\sum_{k=1}^{\infty} c_{k} \cos (2 \pi k x)+d_{k} \sin (2 \pi k x) .
$$

Plugging this back into (76), we obtain

$$
\begin{aligned}
(76)=\int_{0}^{L} \frac{c_{0}}{2} \frac{\sin \left(2 \tilde{\theta}\left(x, E_{1}\right)-2 \tilde{\theta}\left(x, E_{2}\right)\right)}{1+x} d x & +\sum_{k=1}^{\infty} c_{k} \cos (2 \pi k x) \frac{\sin \left(2 \tilde{\theta}\left(x, E_{1}\right)-2 \tilde{\theta}\left(x, E_{2}\right)\right)}{1+x} d x \\
& +\sum_{k=1}^{\infty} d_{k} \sin (2 \pi k x) \frac{\sin \left(2 \tilde{\theta}\left(x, E_{1}\right)-2 \tilde{\theta}\left(x, E_{2}\right)\right)}{1+x} d x .
\end{aligned}
$$

Since $k\left(E_{1}\right), k\left(E_{2}\right) \in\left(0, \frac{\pi}{2}\right)$ or $k\left(E_{1}\right), k\left(E_{2}\right) \in\left(\frac{\pi}{2}, \pi\right)$ depending on either $I \subset\left(a_{n}, c_{n}\right)$ or $I \subset\left(c_{n}, b_{n}\right)$, and $k\left(E_{1}\right) \neq k\left(E_{2}\right)$, we have

$$
0<\left|k\left(E_{1}\right)-k\left(E_{2}\right)\right|<\frac{\pi}{2} .
$$


Since $f \in L^{2}(\mathbb{T})$, on has $\sum c_{k}^{2}+d_{k}^{2}<\infty$. Now the Theorem follows from Lemma 6.4, (77) and (78).

\section{Spectral analysis of Schrödinger operators With EVEntually PERIODIC POTENTIALS}

For $L>0$, let $V_{L}$ be the cut off $V$ up to $L$. More precisely, $V_{L}(x)=V(x)$ for $0 \leq x \leq L$ and $V_{L}(x)=0$ for $x>L$. Let $\mu_{L}$ be the spectral measure corresponding to the operator with potential $V_{L}$.

Theorem 7.1. The following formula hold,

$$
\frac{d \mu_{L}(E)}{d E}=\frac{2}{\pi|W(\bar{\varphi}, \varphi)|} \frac{1}{R^{2}(L, E)}
$$

for $E \in S$.

Proof. Let $Q(z)$ be the transfer matrix of $H_{0}$ from 0 to 1 , that is

$$
Q(z)\left(\begin{array}{c}
\phi(0) \\
\phi^{\prime}(0)
\end{array}\right)=\left(\begin{array}{c}
\phi(1) \\
\phi^{\prime}(1)
\end{array}\right)
$$

for any solution $\phi$ of $-\phi^{\prime \prime}+V_{0} \phi=z \phi$.

Let $z=E+i \varepsilon$ for $E \in S$ and $\varepsilon \geq 0$. Let $k(z)+i \tau(z)$ be such that $2 \cos (k(z)+i \tau(z))=$ $\operatorname{Tr} Q(z)$ with $k(z) \in \mathbb{R}$ and $\tau(z) \in \mathbb{R}$. Thus

$$
\left(e^{-\pi \tau}+e^{\pi \tau}\right) \cos k=\Re \operatorname{Tr} Q(z) ;\left(e^{-\pi \tau}-e^{\pi \tau}\right) \sin k=\Im \operatorname{Tr} Q(z) .
$$

Let us choose the branch so that $k(z) \in(0, \pi)$. Thus

$$
\lim _{\varepsilon \rightarrow 0+} k(E+i \varepsilon)=k(E), \lim _{\varepsilon \rightarrow 0+} \tau(E+i \varepsilon)=0
$$

where $k(E)$ is quasimomentum of $E \in S$.

By the following Lemma 7.2, we have either $\tau(E+i \varepsilon)>0$ for all $\varepsilon>0$ and $E \in S$ or $\tau(E+i \varepsilon)<0$ for all $\varepsilon>0$ and $E \in S$. Without loss of generality, assume $\tau(E+i \varepsilon)<0$.

By the Floquet theory, $-u^{\prime \prime}+V_{0} u=z u$ has a solution with the form:

$$
\varphi(x, z)=J(x, z) e^{-i(k(z)+i \tau(z)) x},
$$

where $J(x, z)$ is 1-periodid? 2 .

Define $\tilde{u}(x, z)=J(x, z) e^{-i(k+i \tau) x}=\varphi(x, z)$ for $x \geq L$ and extend $\tilde{u}(x, z)$ to $0 \leq x \leq$ $L$ by solving equation

$$
-\tilde{u}^{\prime \prime}(x, z)+\left(V_{0}(x)+V_{L}(x)-z\right) \tilde{u}(x, z)=0
$$

for $0 \leq x \leq L$. Since $\gamma(z)<0$, one has $\tilde{u}(x, z) \in L^{2}\left(\mathbb{R}^{+}\right)$. By spectral theory (we refer the readers to [39] and references therein for details), we have

$$
m(z)=\frac{\tilde{u}^{\prime}(0, z)}{\tilde{u}(0, z)},
$$

\footnotetext{
${ }^{2}$ We will show that $\varphi(x, z)$ is an extension of $\varphi(x, E)$ given by (32) and (34) later. This means that there is no confusion even through we use the same notation.
} 
and

$$
\frac{d \mu_{L}}{d E}=\frac{1}{\pi} \lim _{\varepsilon \rightarrow 0+} \Im m(E+i \varepsilon) .
$$

Let $T(z)$ be the transfer matrix of $H_{0}+V_{L}$ from 0 to $L$, that is

$$
T(z)\left(\begin{array}{c}
\phi(0) \\
\phi^{\prime}(0)
\end{array}\right)=\left(\begin{array}{c}
\phi(L) \\
\phi^{\prime}(L)
\end{array}\right)
$$

for any solution $\phi$ of $\left(-D^{2}+V_{0}+V_{L}\right) \phi=z \phi$.

Denote by

$$
T(z)=\left(\begin{array}{ll}
a(z) & b(z) \\
c(z) & d(z)
\end{array}\right) .
$$

Clearly,

$$
\begin{aligned}
\left(\begin{array}{c}
\tilde{u}(0, z) \\
\tilde{u}^{\prime}(0, z)
\end{array}\right) & =\left(\begin{array}{cc}
a(z) & b(z) \\
c(z) & d(z)
\end{array}\right)^{-1}\left(\begin{array}{c}
\tilde{u}(L, z) \\
\tilde{u}^{\prime}(L, z)
\end{array}\right) \\
& =\left(\begin{array}{cc}
d(z) & -b(z) \\
-c(z) & a(z)
\end{array}\right)\left(\begin{array}{c}
\tilde{u}(L, z) \\
\tilde{u}^{\prime}(L, z)
\end{array}\right) .
\end{aligned}
$$

Direct computation implies that (using $a d-b c=1$ )

$$
\begin{aligned}
\lim _{\varepsilon \rightarrow 0+} \Im m(E+i \varepsilon) & =\Im \frac{a \varphi^{\prime}(L, E)-c \varphi(L, E)}{d \varphi(L, E)-b \varphi^{\prime}(L, E)} \\
& =\frac{A_{1} \sin B_{1}}{\left(d-b A_{1} \cos B_{1}\right)^{2}+\left(b A_{1} \sin B_{1}\right)^{2}}
\end{aligned}
$$

where $A_{1}>0$ and $B_{1}$ are defined by

$$
\frac{\varphi^{\prime}(L)}{\varphi(L)}=A_{1} e^{i B_{1}}
$$

Since $\Im m(E+i \varepsilon) \geq 0$ for $\varepsilon \geq 0$, using (82) and (83), one has

$$
\Im\left(\overline{\varphi(x, E)} \varphi^{\prime}(x, E)\right) \geq 0 .
$$

It implies that the restriction of $\varphi(x, z)$ (given by (80) ) on $z \in \mathbb{R}$ coincides with $\varphi(x, E$ ) given by (32) and (34).

It is easy to see that

$$
\begin{aligned}
\left(\begin{array}{c}
u(L, E) \\
u^{\prime}(L, E)
\end{array}\right) & =T(E)\left(\begin{array}{c}
u(0) \\
u^{\prime}(0)
\end{array}\right) \\
& =T(E)\left(\begin{array}{c}
0 \\
1
\end{array}\right)=\left(\begin{array}{l}
b \\
d
\end{array}\right) .
\end{aligned}
$$

Let

$$
\rho(L) \varphi(L)=A_{2} e^{i B_{2}} \text { and } A_{2}>0 .
$$


By (65) and (84), we have

$$
\begin{aligned}
\left(\begin{array}{l}
b \\
d
\end{array}\right) & =\Im A_{2} e^{i B_{2}}\left(\begin{array}{c}
A_{1} e^{i B_{1}} \\
1
\end{array}\right) \\
& =\left(\begin{array}{c}
A_{2} \sin B_{2} \\
A_{1} A_{2} \sin \left(B_{1}+B_{2}\right)
\end{array}\right) .
\end{aligned}
$$

Thus

$$
b=A_{2} \sin B_{2} ; \quad d=A_{1} A_{2} \sin \left(B_{1}+B_{2}\right) .
$$

By (82) and (87),

$$
\lim _{\varepsilon \rightarrow 0+} \Im m(E+i \varepsilon)=\frac{1}{A_{1} A_{2}^{2} \sin B_{1}} .
$$

It is easy to see that (see p.295 in [17])

$$
|\varphi|^{2} \Im \frac{\varphi^{\prime}}{\varphi}=\frac{|W(\bar{\varphi}, \varphi)|}{2}
$$

By (83), (85), (88) and (89),

$$
\begin{aligned}
\lim _{\varepsilon \rightarrow 0+} \Im m(E+i \varepsilon) & =\frac{1}{|\rho(L) \varphi(L)|^{2} \Im\left(\frac{\varphi^{\prime}(L)}{\varphi(L)}\right)} \\
& =\frac{2}{|W(\bar{\varphi}, \varphi)|} \frac{1}{R(L, E)^{2}} .
\end{aligned}
$$

Now the Theorem follows from (81) and (90).

Lemma 7.2. Either $\gamma(E+i \varepsilon)>0$ for all $\varepsilon>0$ and $E \in S$ or $\gamma(E+i \varepsilon)<0$ for all $\varepsilon>0$ and $E \in S$.

Proof. Otherwise, there exist $E \in S$ and $\varepsilon$ such that $\gamma(E+i \varepsilon)=0$. Let $z=E+i \varepsilon$. Therefore, the equation $-u^{\prime \prime}+V_{0} u=z u$ has two linearly independent solutions $\varphi_{1}(x, z)$ and $\varphi_{2}(x, z)$, with the form of

$$
\varphi_{1}(x, z)=J_{1}(x, z) e^{-i k(z) x} \text { and } \varphi_{2}(x, z)=J_{2}(x, z) e^{i k(z) x},
$$

where $J_{1}(x, z)$ and $J_{2}(x, z)$ are 1-periodic. It implies $-u^{\prime \prime}+V_{0} u=z u$ does not have $L^{2}\left(\mathbb{R}^{+}\right)$solution. This is impossible since $\Im z=\varepsilon>0$.

\section{Proof of Theorem 1.5}

In this section, we give the dependence of parameters explicitly except $V_{0}$, since $V_{0}$ is fixed all the time.

Define

$$
\Gamma(E)=\int_{0}^{1} \frac{1}{\left|\gamma^{\prime}(x, E)\right|^{2}} d x .
$$

It easy to see (See [17, Prop.2.1])

$$
\gamma^{\prime}(x, E)=\frac{|W(\bar{\varphi}, \varphi)|}{2|\varphi(x, E)|^{2}} .
$$

Let $L=\epsilon^{-1-\sigma}$. Let $C_{1}=C_{1}(B, I)$, which will be determined later. 
We say a subset $A \subset I$ is $(\epsilon, N)$ separate, if the following two conditions hold:

For any $E \in A$,

$$
\left|\int_{0}^{L} V(x) \frac{\sin 2 \theta(x, E)}{\gamma^{\prime}(x, E)} d x\right| \geq(1-\beta) C_{1}(B, I) \log \epsilon^{-1} .
$$

For any $E_{1}, E_{2} \in A$ and $E_{1} \neq E_{2}$,

$$
\left|k\left(E_{1}\right)-k\left(E_{2}\right)\right| \geq \epsilon^{1 / N^{2}} .
$$

Lemma 8.1. [16, Lemma 4.4] Let $\left\{e_{i}\right\}_{i=1}^{N}$ be a set of unit vectors in a Hilbert space $\mathcal{H}$ so that

$$
\alpha=N \sup _{i \neq j}\left|\left\langle e_{i}, e_{j}\right\rangle\right|<1
$$

Then for any $g \in \mathcal{H}$,

$$
\sum_{i=1}^{N}\left|\left\langle g, e_{i}\right\rangle\right|^{2} \leq(1+\alpha)\|g\|^{2} .
$$

Theorem 8.2. There exists $\epsilon_{1}(B, I, \sigma, \beta)>0$ and $C(B, I, \sigma, \beta)$ such that for any $\epsilon<\epsilon_{1}$ and $N \geq C(B, I, \sigma, \beta)$, the $(\epsilon, N)$ separate set $A$ satisfies $\# A \leq N$.

Proof. We consider the Hilbert space

$$
\mathcal{H}=L^{2}((0, L),(1+x) d x) .
$$

In $\mathcal{H}$, by (62) we have

$$
\|V\|_{\mathcal{H}}^{2} \leq B^{2} \log (1+L)
$$

Let

$$
e_{i}(x)=\frac{1}{\sqrt{A_{i}}} \frac{\sin 2 \theta\left(x, E_{i}\right)}{\gamma^{\prime}\left(x, E_{i}\right)(1+x)} \chi_{[0, L]}(x),
$$

where $A_{i}$ is chosen so that $e_{i}$ is an unit vector in $\mathcal{H}$. We have the following estimate,

$$
\begin{aligned}
A_{i} & =\int_{0}^{B_{j}} \frac{\sin ^{2} 2 \theta\left(x, E_{i}\right)}{\left|\gamma^{\prime}\left(x, E_{i}\right)\right|^{2}(1+x)} d x \\
& =\int_{0}^{L} \frac{1}{2\left|\gamma^{\prime}\left(x, E_{i}\right)\right|^{2}(1+x)} d x-\int_{0}^{L} \frac{\cos 4 \theta\left(x, E_{i}\right)}{\left|\gamma^{\prime}\left(x, E_{i}\right)\right|^{2}(1+x)} d x .
\end{aligned}
$$

By (69), one has

$$
\left|\int_{0}^{L} \frac{\cos 4 \theta\left(x, E_{i}\right)}{\left|\gamma^{\prime}\left(x, E_{i}\right)\right|^{2}(1+x)} d x\right|=O(1)
$$

Direct computation shows that

$$
\begin{aligned}
\int_{0}^{L} \frac{1}{\left|\gamma^{\prime}\left(x, E_{i}\right)\right|^{2}(1+x)} d x & =O(1)+\sum_{n=0}^{L-1} \int_{n}^{n+1} \frac{1}{\left|\gamma^{\prime}\left(x, E_{i}\right)\right|^{2}(1+n)} d x \\
& =O(1)+\Gamma\left(E_{i}\right) \log L .
\end{aligned}
$$


By (97), (98) and (99), we have

$$
A_{i}=\frac{1}{2} \Gamma\left(E_{i}\right) \log L+O(1) .
$$

We should mention that $O(1)$ in (98), (99) and (100) only depends on $B$ and $I$.

By (70) and (96), we have

$$
\mid\left\langle e_{i}, e_{j}\right\rangle \leq \frac{2}{1+\sigma} C(I, B) N^{-2}+\frac{C(I, B)}{\log \epsilon^{-1}} .
$$

The first condition (92) implies

$$
\left|\left\langle V, e_{i}\right\rangle\right|^{2} \geq \frac{C_{1}^{2}}{1+\sigma} \log \epsilon^{-1}-C(I, B) .
$$

By (94) and (101), one has

$$
\sum_{i=1}^{N}\left|\left\langle V, e_{i}\right\rangle_{\mathcal{H}}\right|^{2} \leq\left(1+\frac{2}{1+\sigma} C(I, B) N^{-1}+\frac{N C(I, B)}{\log \epsilon^{-1}}\right)\|V\|_{\mathcal{H}} .
$$

By (102), (95) and (103), we have

$N\left(\frac{C_{1}^{2}(1-\beta)^{2}}{1+\sigma} \log \epsilon^{-1}-C(I, B)\right) \leq\left(1+\frac{2}{1+\sigma} C(I, B) N^{-1}+\frac{N C(I, B)}{\log \epsilon^{-1}}\right) B^{2}(1+\sigma) \log \epsilon^{-1}$.

This implies the Theorem.

Proof of Theorem 1.5. Once we have Theorems 7.1 and 8.2 , Theorem 1.5 can be proved by the arguments in [15] (also see [22]). We omit the details here.

\section{Appendix A.}

Proof of (40). By (36) and (40), it suffices to show that

$$
\frac{i}{2 \Im\left(\varphi \bar{\varphi}^{\prime}\right)} V(x) \bar{\varphi}^{2} \exp (-2 i p)=\frac{-i V}{2 \Re \phi^{\prime}} e^{-i h}
$$

By the definition, one has

$$
\phi^{\prime}=-i \frac{\varphi^{\prime}}{\varphi}
$$

Direct computations imply

$$
\frac{|\varphi|^{2}}{\Im\left(\varphi \bar{\varphi}^{\prime}\right)}=-\frac{1}{\Re \phi^{\prime}} .
$$


By the definitions of $h$ and $p$, we have

$$
\begin{aligned}
\frac{i V}{2 \Re \phi^{\prime}} e^{-i h} & =\frac{i V}{2 \Re \phi^{\prime}} \exp \left(-i 2 \Re \phi+i \int_{0}^{x} \frac{V(t)}{\Re \phi^{\prime}(t, E)} d t\right) \\
& =-i V \frac{|\varphi|^{2}}{2 \Im\left(\varphi \bar{\varphi}^{\prime}\right)} \exp (-i 2 \Re \phi) \exp \left(-i \int_{0}^{x} \frac{V(t)|\varphi(t, E)|^{2}}{\Im\left(\varphi \bar{\varphi}^{\prime}\right)} d t\right) \\
& =-i V \frac{|\varphi|^{2}}{2 \Im\left(\varphi \bar{\varphi}^{\prime}\right)} \exp (-i 2 \Re \phi) \exp (-2 i p) \\
& =-i V \frac{\bar{\varphi}^{2}}{2 \Im\left(\varphi \bar{\varphi}^{\prime}\right)} \exp (-2 i p) .
\end{aligned}
$$

It implies (104) and hence (40).

Proof of (31). Denote by

$$
f_{k}=\int_{k-1}^{k}|f(x)| d x
$$

for $k \in \mathbb{Z}_{+}$. Then

$$
\|f\|_{\ell^{2}\left(L^{1}\right)}^{2}=\sum_{k=1}^{\infty} f_{k}^{2} .
$$

Direct computations imply

$$
\begin{aligned}
\int_{\mathbb{R}_{+}^{2}} \frac{|f(x) f(y)|}{1+|x-y|^{2}} d x d y & =O(1) \sum_{m=1}^{\infty} \sum_{n=1}^{\infty} \frac{f_{m} f_{n}}{1+|m-n|^{2}} \\
& =O(1) \sum_{n=1}^{\infty}\left|f_{n}\right|^{2}
\end{aligned}
$$

where the second equality holds by Young's convolution inequality. Now (31) follows from (106) and (107).

\section{ACKNOWLEDGMENTS}

I would like to thank Barry Simon and Gunter Stolz for some useful discussion. This research was supported by NSF DMS-1700314/2015683, DMS-2000345 and the Southeastern Conference (SEC) Faculty Travel Grant 2020-2021.

\section{REFERENCES}

[1] M. Christ and A. Kiselev. Absolutely continuous spectrum for one-dimensional Schrödinger operators with slowly decaying potentials: some optimal results. $J$. Amer. Math. Soc., 11(4):771-797, 1998.

[2] M. Christ and A. Kiselev. Maximal functions associated to filtrations. J. Funct. Anal., 179(2):409-425, 2001.

[3] M. Christ and A. Kiselev. WKB and spectral analysis of one-dimensional Schrödinger operators with slowly varying potentials. Comm. Math. Phys., 218(2):245-262, 2001. 
[4] M. Christ and A. Kiselev. WKB asymptotic behavior of almost all generalized eigenfunctions for one-dimensional Schrödinger operators with slowly decaying potentials. J. Funct. Anal., 179(2):426-447, 2001.

[5] P. Deift and R. Killip. On the absolutely continuous spectrum of one-dimensional Schrödinger operators with square summable potentials. Comm. Math. Phys., 203(2):341-347, 1999.

[6] S. A. Denisov and A. Kiselev. Spectral properties of Schrödinger operators with decaying potentials. In Spectral theory and mathematical physics: a Festschrift in honor of Barry Simon's 60th birthday, volume 76 of Proc. Sympos. Pure Math., pages 565-589. Amer. Math. Soc., Providence, RI, 2007.

[7] J. D. Dollard and C. N. Friedman. Asymptotic behavior of solutions of linear ordinary differential equations, 1978.

[8] S. Jitomirskaya and W. Liu. Noncompact complete Riemannian manifolds with dense eigenvalues embedded in the essential spectrum of the Laplacian. Geom. Funct. Anal., 29(1):238-257, 2019.

[9] T. Kato. Growth properties of solutions of the reduced wave equation with a variable coefficient. Comm. Pure Appl. Math., 12:403-425, 1959.

[10] R. Killip. Perturbations of one-dimensional Schrödinger operators preserving the absolutely continuous spectrum. Int. Math. Res. Not., (38):2029-2061, 2002.

[11] R. Killip. Spectral theory via sum rules. In Spectral theory and mathematical physics: a Festschrift in honor of Barry Simon's 60th birthday, volume 76 of Proc. Sympos. Pure Math., pages 907-930. Amer. Math. Soc., Providence, RI, 2007.

[12] A. Kim and A. Kiselev. Absolutely continuous spectrum of discrete Schrödinger operators with slowly oscillating potentials. Math. Nachr., 282(4):552-568, 2009.

[13] A. Kiselev. Absolutely continuous spectrum of one-dimensional Schrödinger operators and Jacobi matrices with slowly decreasing potentials. Comm. Math. Phys., 179(2):377-400, 1996.

[14] A. Kiselev. Stability of the absolutely continuous spectrum of the Schrödinger equation under slowly decaying perturbations and a.e. convergence of integral operators. Duke Math. J., 94(3):619-646, 1998.

[15] A. Kiselev. Imbedded singular continuous spectrum for Schrödinger operators. $J$. Amer. Math. Soc., 18(3):571-603, 2005.

[16] A. Kiselev, Y. Last, and B. Simon. Modified Prüfer and EFGP transforms and the spectral analysis of one-dimensional Schrödinger operators. Comm. Math. Phys., 194(1):1-45, 1998.

[17] A. Kiselev, C. Remling, and B. Simon. Effective perturbation methods for onedimensional Schrödinger operators. J. Differential Equations, 151(2):290-312, 1999.

[18] T. Kriecherbauer and C. Remling. Finite gap potentials and WKB asymptotics for one-dimensional Schrödinger operators. Comm. Math. Phys., 223(2):409-435, 2001.

[19] P. Kuchment. An overview of periodic elliptic operators. Bull. Amer. Math. Soc. (N.S.), 53(3):343-414, 2016. 
[20] P. Kurasov and S. Naboko. Wigner-von Neumann perturbations of a periodic potential: spectral singularities in bands. Math. Proc. Cambridge Philos. Soc., 142(1):161-183, 2007.

[21] Y. Last and B. Simon. The essential spectrum of Schrödinger, Jacobi, and CMV operators. J. Anal. Math., 98:183-220, 2006.

[22] W. Liu. Absence of singular continuous spectrum for perturbed discrete Schrödinger operators. J. Math. Anal. Appl., 472(2):1420-1429, 2019.

[23] W. Liu. The asymptotical behaviour of embedded eigenvalues for perturbed periodic operators. Pure Appl. Funct. Anal., 4(3):589-602, 2019.

[24] W. Liu. Criteria for eigenvalues embedded into the absolutely continuous spectrum of perturbed Stark type operators. J. Funct. Anal., 276(9):2936-2967, 2019.

[25] W. Liu and D. C. Ong. Sharp spectral transition for eigenvalues embedded into the spectral bands of perturbed periodic operators. J. Anal. Math. to appear.

[26] M. Lukic. Schrödinger operators with slowly decaying Wigner-von Neumann type potentials. J. Spectr. Theory, 3(2):147-169, 2013.

[27] M. Lukic. Square-summable variation and absolutely continuous spectrum. $J$. Spectr. Theory, 4(4):815-840, 2014.

[28] M. Lukic and D. C. Ong. Wigner-von Neumann type perturbations of periodic Schrödinger operators. Trans. Amer. Math. Soc., 367(1):707-724, 2015.

[29] C. Muscalu, T. Tao, and C. Thiele. A counterexample to a multilinear endpoint question of Christ and Kiselev. Math. Res. Lett., 10(2-3):237-246, 2003.

[30] S. Naboko and S. Simonov. Zeroes of the spectral density of the periodic Schrödinger operator with Wigner-von Neumann potential. Math. Proc. Cambridge Philos. Soc., 153(1):33-58, 2012.

[31] S. N. Naboko. On the dense point spectrum of Schrödinger and Dirac operators. Teoret. Mat. Fiz., 68(1):18-28, 1986.

[32] C. Remling. The absolutely continuous spectrum of one-dimensional Schrödinger operators with decaying potentials. Comm. Math. Phys., 193(1):151-170, 1998.

[33] C. Remling. Bounds on embedded singular spectrum for one-dimensional Schrödinger operators. Proc. Amer. Math. Soc., 128(1):161-171, 2000.

[34] C. Remling. Schrödinger operators with decaying potentials: some counterexamples. Duke Math. J., 105(3):463-496, 2000.

[35] F. S. Rofe-Beketov. A finiteness test for the number of discrete levels which can be introduced into the gaps of the continuous spectrum by perturbations of a periodic potential. Dokl. Akad. Nauk SSSR, 156:515-518, 1964.

[36] B. Simon. Bounded eigenfunctions and absolutely continuous spectra for onedimensional Schrödinger operators. Proc. Amer. Math. Soc., 124(11):3361-3369, 1996.

[37] B. Simon. Some Schrödinger operators with dense point spectrum. Proc. Amer. Math. Soc., 125(1):203-208, 1997.

[38] B. Simon. Schrödinger operators in the twenty-first century. In Mathematical physics 2000, pages 283-288. Imp. Coll. Press, London, 2000.

[39] B. Simon. Analogs of the $m$-function in the theory of orthogonal polynomials on the unit circle. J. Comput. Appl. Math., 171(1-2):411-424, 2004. 
[40] G. Stolz. On the absolutely continuous spectrum of perturbed Sturm-Liouville operators. J. Reine Angew. Math., 416:1-23, 1991.

[41] G. Stolz. Bounded solutions and absolute continuity of Sturm-Liouville operators. J. Math. Anal. Appl., 169(1):210-228, 1992.

[42] J. von Neumann and E. Wigner. Uber merkwürdige diskrete Eigenwerte; Uber das Verhalten von Eigenwerten bei adiabatischen Prozessen Phys. Zeitschrift, 30:467470, 1929.

Department of Mathematics, Texas A\&M University, College Station, TX 77843-3368, USA

E-mail address: liuwencai1226@gmail.com 